
\title{
6

\section{Peroral Pancreatoscopy with Videoscopy and Narrow-Band Imaging in Intraductal Papillary Mucinous Neoplasms with Dilatation of the Main Pancreatic Duct}

\author{
Yui Kishimoto, Naoki Okano, Ken Ito, Kensuke Takuma, Seiichi Hara, Susumu Iwasaki, Kensuke Yoshimoto, Yuto Yamada, Koji \\ Watanabe, Yusuke Kimura, Hiroki Nakagawa, and Yoshinori Igarashi \\ Division of Gastroenterology and Hepatology, Department of Internal Medicine, Toho University Omori Medical Center, Tokyo, Japan
}

Background/Aims: Endoscopic evaluation of intraductal papillary mucinous neoplasms (IPMNs) is useful in determining whether the lesions are benign or malignant. This study aimed to examine the usefulness of peroral pancreatoscopy (POPS) in determining the prognosis of IPMNs.

Methods: POPS with videoscopy was performed using the mother-baby scope technique. After surgery, computed tomography/ magnetic resonance cholangiopancreatography or ultrasonography and blood tests were performed every 6 months during the follow-up.

Results: A total of 39 patients with main pancreatic duct (MPD)-type IPMNs underwent POPS using a videoscope, and the protrusions in the MPD were observed in 36 patients. The sensitivity and specificity of cytology/biopsy performed at the time of POPS were $85 \%$ and $87.5 \%$, respectively. Of 19 patients who underwent surgery, 18 (95\%) patients had negative surgical margins and 1 (5\%) patient had a positive margin.

Conclusions: In IPMNs with dilatation of the MPD, POPS is considered effective if the lesions can be directly observed. The diagnosis of benign and malignant lesions is possible depending on the degree of lesion elevation. However, in some cases, slightly elevated lesions may increase in size during the follow-up or multiple lesions may be simultaneously present; therefore, careful follow-up is necessary. Clin Endosc 2022;55:270-278

Key Words: Endoscopic diagnosis; Image-enhanced endoscopy; Intraductal papillary mucinous neoplasm; Peroral videopancreatoscopy

\section{INTRODUCTION}

Intraductal papillary mucinous neoplasms (IPMNs) can be classified as either main pancreatic duct (MPD)-type IPMNs (MD-IPMNs) or branched duct-type IPMNs (BD-IPMNs)

Received: February 14, 2021 Revised: July 17, 2021

Accepted: July 27, 2021

Correspondence: Yui Kishimoto

Division of Gastroenterology and Hepatology, Department of Internal Medicine, Toho University Omori Medical Center, 6-11-1, Omorinishi, Ohta-ku, Tokyo 143-8541, Japan

Tel: +81-3-3762-4151, Fax: +81-3-3763-8542, E-mail: yuui.kishimoto@med. toho-u.ac.jp

ORCID: https://orcid.org/0000-0001-5040-2938

(c) This is an Open Access article distributed under the terms of the Creative Commons Attribution Non-Commercial License (http://creativecommons.org/ licenses/by-nc/3.0) which permits unrestricted non-commercial use, distribution, and reproduction in any medium, provided the original work is properly cited. according to the tumor site and mode of progression. The incidence of malignancy differs between the two types of IPMNs; therefore, the classification of the type of neoplasm is useful in determining the treatment course. ${ }^{1-6}$ However, imaging diagnosis and tissue diagnosis do not always correlate. ${ }^{7}$

Endoscopic diagnosis has achieved remarkable progress owing to improvements in videoscopy and observation equipment. ${ }^{8}$ In recent years, small-diameter videoscopes have been developed, ${ }^{9,10}$ enabling the examination of the interior of the MPD. Thus, videoscopic imaging has considerably improved and its clinical application is increasing. ${ }^{11-19}$ Furthermore, a clearer image can be obtained when using a videoscope. ${ }^{20-23}$ IPMNs are pancreatic intraductal tumors characterized by papillary proliferation of mucin-producing epithelial cells and include various lesions, ranging from hyperplastic changes to adenocarcinomas. By observing the MPD using peroral 
pancreatoscopy (POPS), it is possible to directly inspect the elevated lesions and differentiate between benign and malignant types based on the lesion morphology. ${ }^{24-28}$

Since 2003, we have performed image-enhanced endoscopy (IEE) with POPS using a videoscope. With narrow-band imaging (NBI), small protrusions and vessels can be clearly observed within the pancreatic duct for endoscopic diagnosis, as previously reported. ${ }^{29}$

This study aimed to examine the usefulness of POPS in determining the prognosis of IPMNs.

\section{MATERIALS AND METHODS}

\section{Subjects}

This single-center retrospective study enrolled 39 patients with IPMNs who were admitted to Toho University Medical Center Omori Hospital between April 2003 and September 2018. Imaging studies (e.g., computed tomography, ultrasonography, and magnetic resonance cholangiopancreatography) were performed, followed by POPS, in 39 cases. All patients had dilatation of the main papilla and MPD, as observed during endoscopic retrograde cholangiopancreatography (ERCP). This study included 27 male and 12 female patients with an average age of 68.4 years. Patients who could not undergo endoscopy because of chronic respiratory failure, heart failure, or other reasons were excluded from the study.

\section{Methods}

We performed POPS during ERCP using the mother-baby scope method with injection of saline. The mother scope used in the procedure was Olympus TJF-240 or TJF-260V (Olympus, Tokyo, Japan), and the baby scope was CHF-BP260, CHF-B260, or CHF-B290 (Olympus, Tokyo, Japan).

The EVIS240/EVIS260SL/EVISCLV-290SL system (Olympus, Tokyo, Japan) was used as the observation equipment. EVIS260SL was used from July 2007 to February 2013. Thereafter, from March 2013, EVISCLV-290SL was used for observation with IEE using NBI. Still and moving images were recorded on a hard disk using an electronic filing system.

The results of insertion of the baby scope into the MPD, characteristics of the target lesion site, morphology of protrusions, preoperative morphology, and histopathologic diagnosis were examined. The morphology of protrusions was classified into the following four types: sessile morphology (Is) (Fig. $1 \mathrm{~A}$ and B), semipedunculated morphology (Isp) (Fig. 1C and D), villous morphology (Vil) (Fig. 2A and B ), and vegetative morphology (Veg) (Fig. 2C and D ). ${ }^{24,26}$ On the basis of preoperative pathologic findings, cytology was classified into classes 0 , I, II, IIIa, IIIb, IV, and V and biopsy results were classified as follows: no tissue, hyperplasia, mild atypia, adenoma, and adenocarcinoma.

Patients in whom malignancy was suspected on the basis of the morphology of protrusions and cytology/biopsy findings underwent surgical resection. The preoperative pathologic diagnosis and the histologic interpretations of the resected specimens were performed by one or two experienced pathologists.

This study was approved by the Ethics Committee of Toho University Medical Center Omori Hospital (M20248). Details of the study are available on the Institute's website and consent was obtained on an opt-out basis.

\section{Statistical analysis}

All clinical and endoscopic variables were obtained from the database. For the purpose of analysis, cytologic specimens were categorized as positive (classes IIIb, IV, and V) or negative (classes I, II, and IIIa). Similarly, biopsy specimens were categorized as positive (adenocarcinoma) or negative (no tissue, hyperplasia, mild atypia, or adenoma). Sensitivity, specificity, and negative predictive values were calculated on the basis of the positive and negative findings of cytology and biopsy.

The long-term prognosis of all patients was examined using the Kaplan-Meier method and log-rank test, and the prognoses of benign and malignant lesions after endoscopic diagnosis were compared.

Statistical significance was set at $p<0.05$. All statistical analyses were performed using JMP ${ }^{\circledR}$ software (SAS Institute Inc., Cary, NC, USA).

\section{RESULTS}

\section{POPS and observation success rates}

We inserted the baby scope into the MPD in all 39 patients (Fig. 3). Protrusions in the MPD were observed using POPS in 36 patients (93\%), and the observation was unsuccessful in 3 patients. The reasons for failure of the procedure were strong bending of the MPD in one patient and BD-IPMNs in two patients. These two patients were not included in the determination of prognosis. In one patient, the baby scope could not pass through the pancreatic neck because of the strong bending of the MPD at the transitional part of the pancreatic head, whereas branch-type IPMNs were observed in two patients. No lesions were observed in the MPD. From April 2004, the other 36 patients were observed with IEE using NBI. Post-ER$\mathrm{CP}$ pancreatitis was not observed in the present study.

\section{Endoscopic findings}

The examination revealed 7 cases of the Is morphology, 10 cases of the Isp morphology, 10 cases of the Vil morphology, 


\section{C clinical endoscopr}

Sessile morphology (Is)

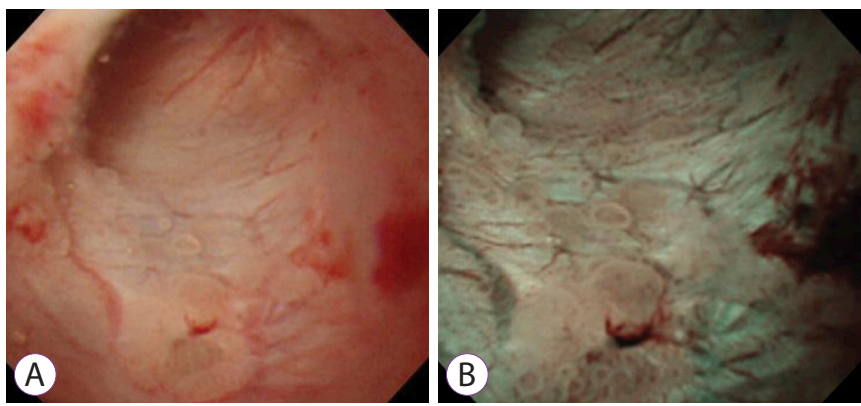

Semipedunculated morphology (Isp)

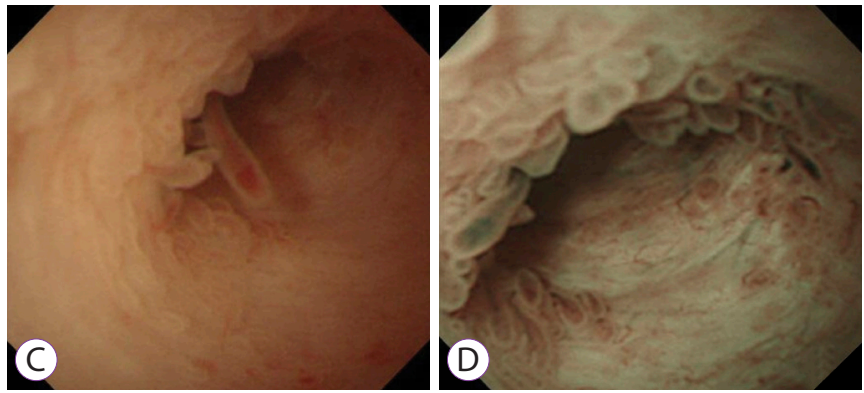

Fig. 1. Peroral pancreatoscopy images of intraductal papillary mucinous neoplasms. (A,B) White-light imaging and narrow-band imaging of a sessile morpholgy. (C, D) White-light imaging and narrow-band imaging of a semipedunculated morphology.

Villous morphology (Vil)
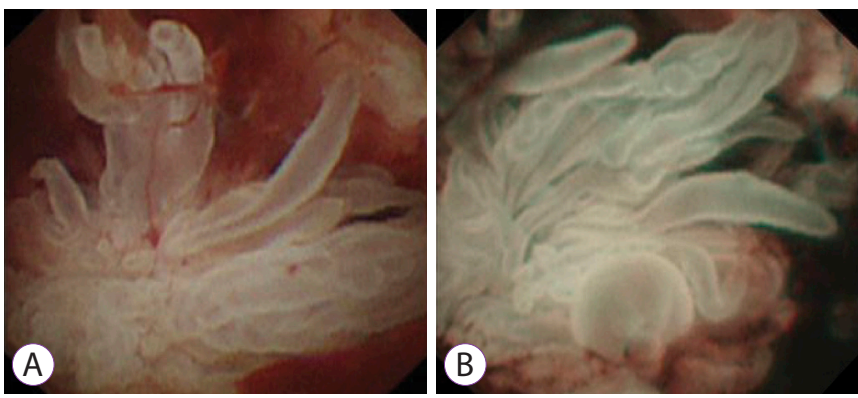

Vegetative morphology (Veg)

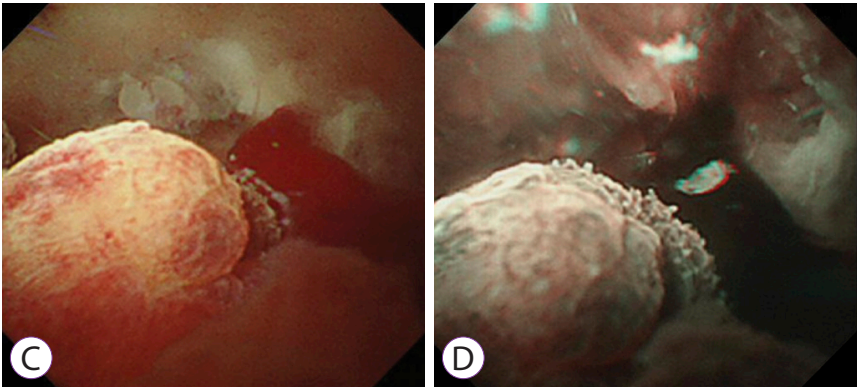

Fig. 2. Peroral pancreatoscopy images of intraductal papillary mucinous neoplasms. (A, B) White-light imaging and narrow-band imaging of a villous morphology. (C, D) White-light imaging and narrow-band imaging of a vegetative morphology. 
and 9 cases of the Veg morphology (Table 1). Cytology was performed in 33 cases, and biopsy was performed in 8 cases; both cytology and biopsy were performed in 7 cases.

\section{Cytologic diagnosis}

On the basis of the cytologic findings, the Is morphology was class I in one case, class II in one case, class IIIa in three cases, and class 0 (no tissue) in two cases.

The Isp morphology was class I in none of the cases, class II in one case, class IIIa in four cases, class IIIb in two cases, class IV in one case, and class 0 (no tissue) in two cases. The Vil morphology was class II in one case, class IIIa in three cases, class IIIb in four cases, and class V in two cases. The Veg morphology was class IIIa in five cases, class IIIb in one case, class IV in one case, and class $\mathrm{V}$ in two cases.

\section{Biopsy findings}

One patient with the Isp morphology was diagnosed with hyperplasia; three patients with the Vil morphology were diagnosed with adenocarcinoma; one patient with the Vil morphology was diagnosed with mild atypia; and one and two patients with the Veg morphology were diagnosed with lowgrade adenoma and carcinoma, respectively.

\section{Accuracy of POPS and pathologic findings}

Cytology/biopsy had a diagnostic sensitivity of $85 \%$ and specificity of $87.5 \%$. The positive and negative predictive values were $89.5 \%$ and $82.4 \%$, respectively. Of the 19 patients who underwent surgery, 18 (95\%) patients had negative margins and $1(5 \%)$ patient had a positive margin.

\section{Endoscopic morphology and clinical course}

All seven patients with the Is morphology were followed up (mean observation period, 81 months). The pathology of six patients remained unchanged. In one patient, the cyst increased in size and POPS reexamination showed a change to the Vil morphology. The cancer worsened, and the patient died after refusing surgical intervention (at 113 months) (Fig. 4).

Of the 10 patients with the Isp morphology, 7 patients were followed up and all of them survived (mean observation period, 107 months). Of them, two patients had severe atypia. One patient had multiple lesions consisting of mild atypia in the head of the pancreas and invasive adenocarcinoma in the body and tail of the pancreas. The remaining patient had gastric cancer and IPMN (moderate atypia) simultaneously and underwent gastropancreatoduodenectomy (Fig. 5).

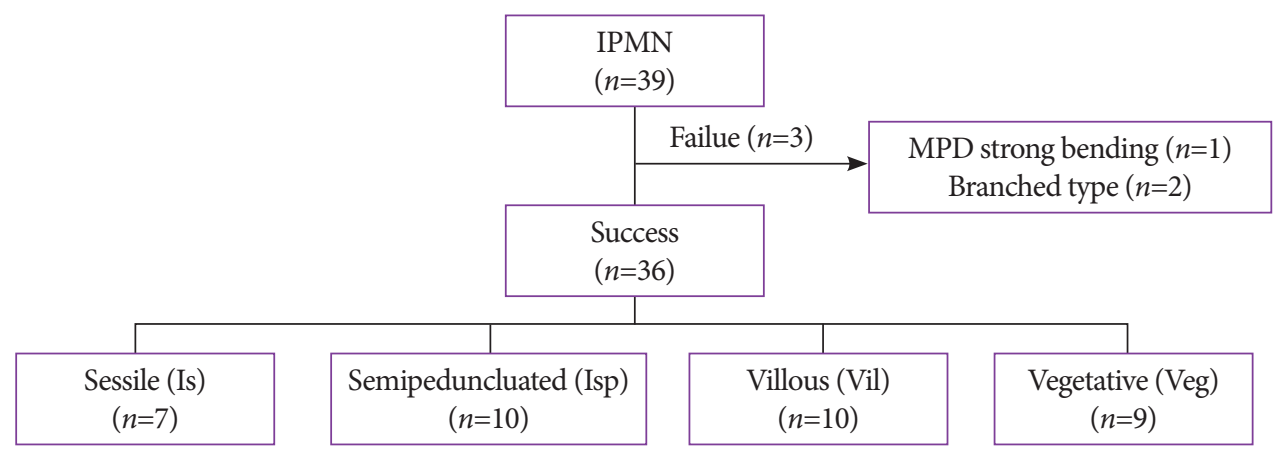

Fig. 3. Study flowchart. IPMN, intraductal papillary mucinous neoplasm; MPD, main pancreatic duct; POPS, peroral pancreatoscopy

Table 1. Endoscopic Classification of Protrusions

\begin{tabular}{lcccc} 
& Is $(\boldsymbol{n}=7)$ & Isp $(\boldsymbol{n}=\mathbf{1 0})$ & Vil $(\boldsymbol{n}=\mathbf{1 0})$ & Veg $(\boldsymbol{n}=\mathbf{9})$ \\
\hline Sex: M/F & $3 / 4$ & $10 / 0$ & $6 / 4$ & $6 / 3$ \\
Average age (years) & 74.2 & 65.8 & 67.9 & 70.7 \\
Surgery +/- & $0 / 7$ & $6 / 4$ & $6 / 4$ & $7 / 2$ \\
Pathologic diagnosis after surgery & NA & Adenocarcinoma: 3, atypia: 3 & Adenocarcinoma: 5, atypia: 1 & Adenocarcinoma: 7 \\
\hline
\end{tabular}

F, female; Is, sessile morphology; Isp, semipedunculated morphology; M, male; NA, not applicable; Veg, vegetative morphology; Vil, villous morphology. 
Among the 10 patients with the Isp morphology, 6 underwent surgery ( 3 patients with cancer and 3 patients with benign lesions). The reasons for surgery were cytologic diagnosis of class IIIb in three patients, diagnosis of class IVb in one patient, simultaneous resection of gastric cancer in one patient, and repetitive pancreatitis in one patient (Fig. 5).

During the follow-up, three deaths occurred. One death was due to cancer (at 45 months), and two deaths were due to other diseases (at 41 and 84 months) (Fig. 5).

Among the 10 patients with the Vil morphology, 6 patients underwent surgery. Of these six patients, two had invasive adenocarcinoma, one had microinvasion, two had noninvasive adenocarcinoma, and one had severe atypia. After the surgery, four patients were alive and two patients died (one patient with cancer and one patient without cancer). The four patients who did not undergo surgery were followed up without treatment. Of them, one patient was alive and three patients died (two patients died of cancer and one patient died of respiratory failure) (Fig. 6).

Surgery was performed in seven of the nine patients with the Veg morphology (seven patients with adenocarcinoma)
(Fig. 7). Of the seven patients who underwent surgery, six survived with an average observation period of 85 months, and one patient died after postoperative recurrence (at 53 months). The two patients who refused surgery showed an increased tendency for malignant transformation; however, there was no invasion to other organs, and they were still alive during the study period.

In the comparison between the Is + Isp and Vil + Veg groups, the Kaplan-Meier method showed a 50\% survival period of 239 months in the Is + Isp group and 133 months in the Vil + Veg group, indicating that the overall survival of the Is + Isp group was longer than that of the Vil + Veg group; however, no significant difference was observed $(p=0.1473)$ (Fig. 8). Long-term prognosis (excluding deaths from other illnesses) was classified as benign or malignant according to the preoperative endoscopic diagnosis. The overall survival tended to be longer in the benign group than in the malignant group; however, no significant difference was observed $(p=0.1114)$ (Fig. 9). Analyses with $\chi^{2}$ testing were not possible because of the small number of patients in this study.

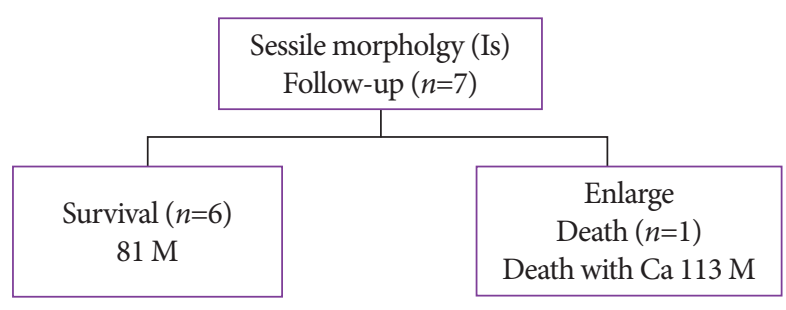

Fig. 4. Prognosis of sessile morphology (Is): endoscopic morphology and clinical course. Ca, Adenocrcinoma. M, months.

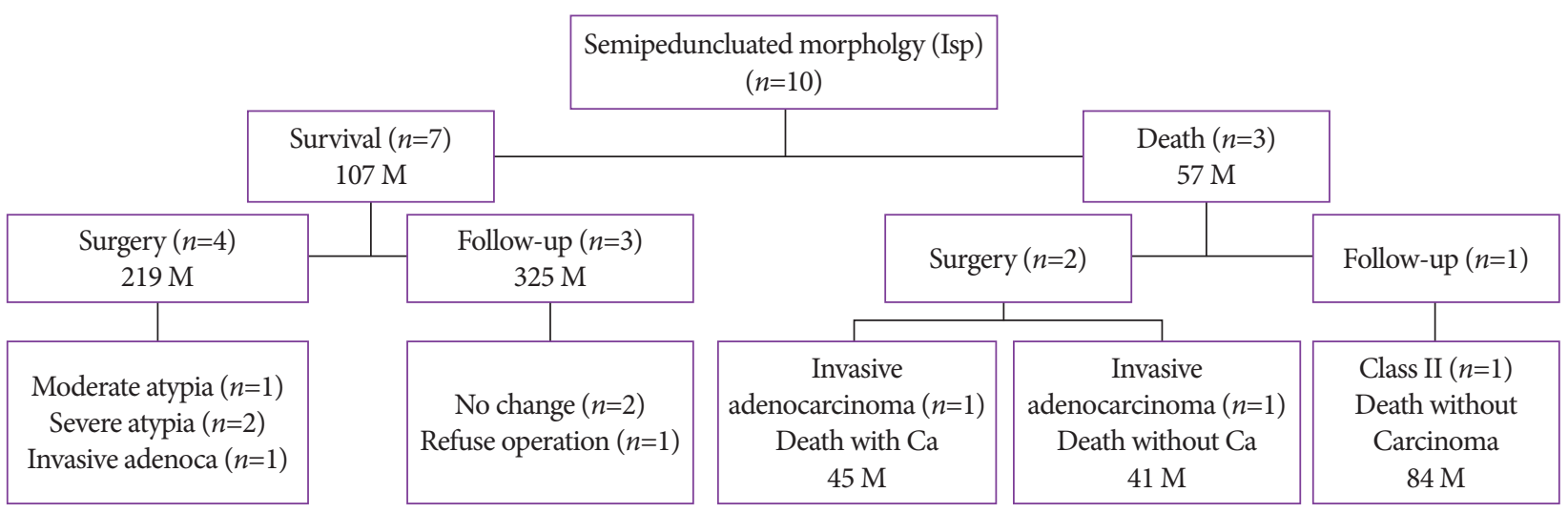

Fig. 5. Prognosis of semipedunculated morphology (Isp). M, months. 


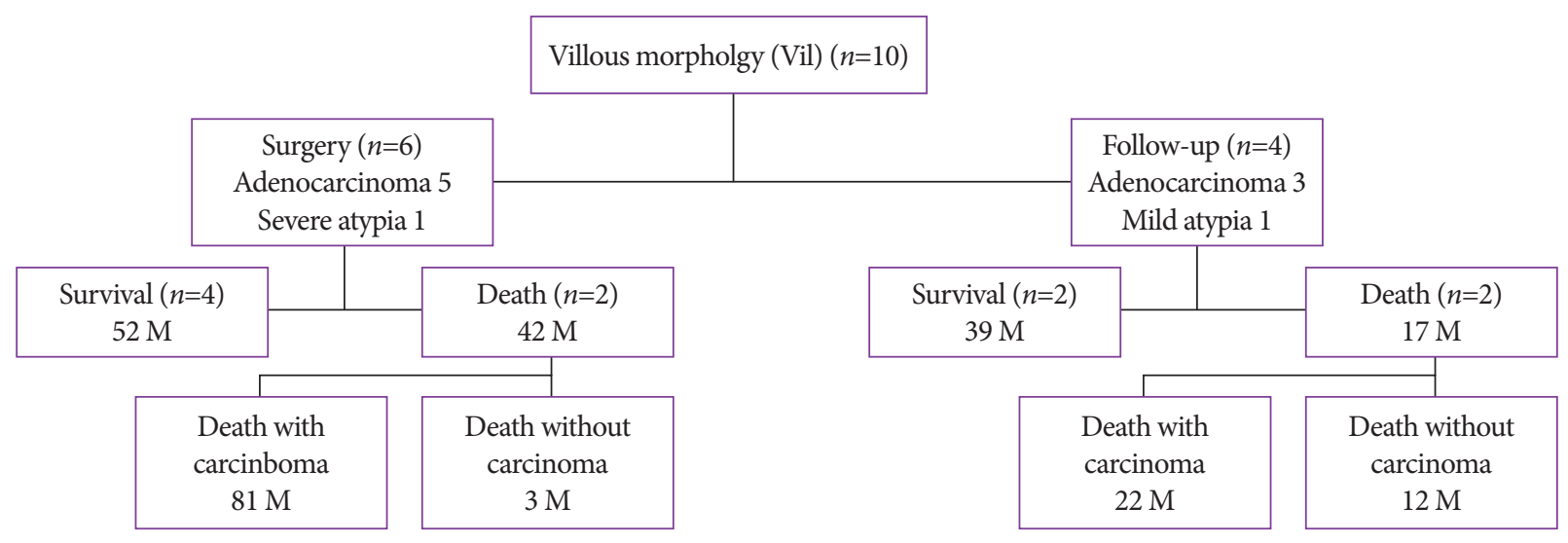

Fig. 6. Prognosis of villous morphology (Vil). M, months.

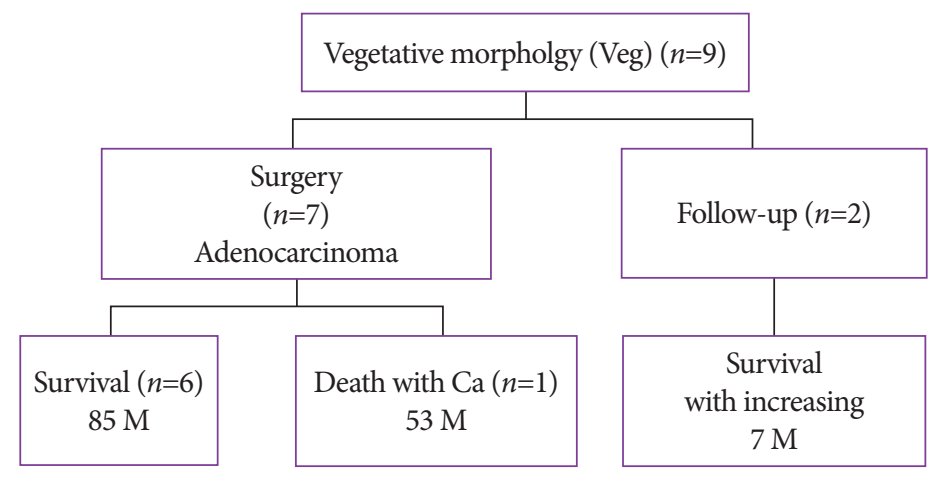

CA: Adenocrcinoma

Fig. 7. Prognosis of vegetative morphology (Veg). Ca, Adenocrcinoma; M, months.

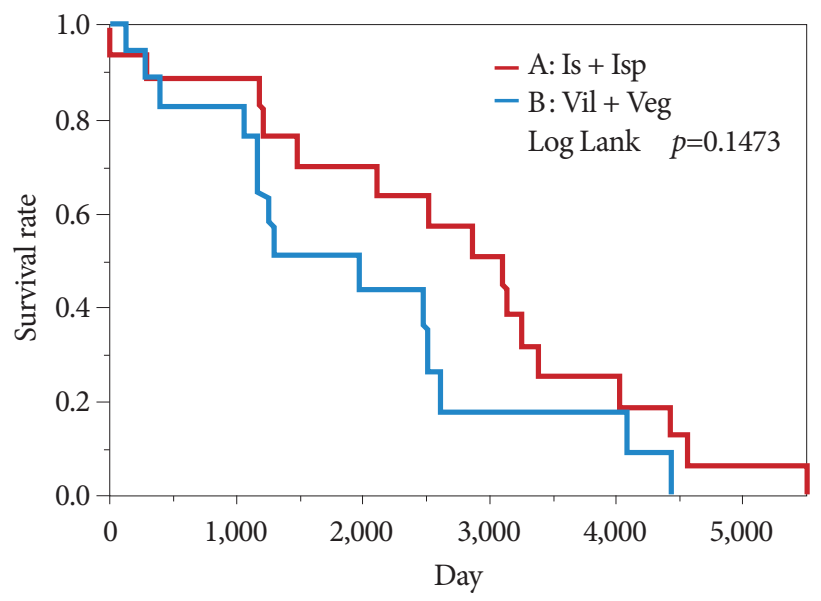

Fig. 8. Prognosis of sessile morphology (Is) + semipedunculated morphology (Isp) versus villous morphology (Vil) + vegetative morphology (Veg).

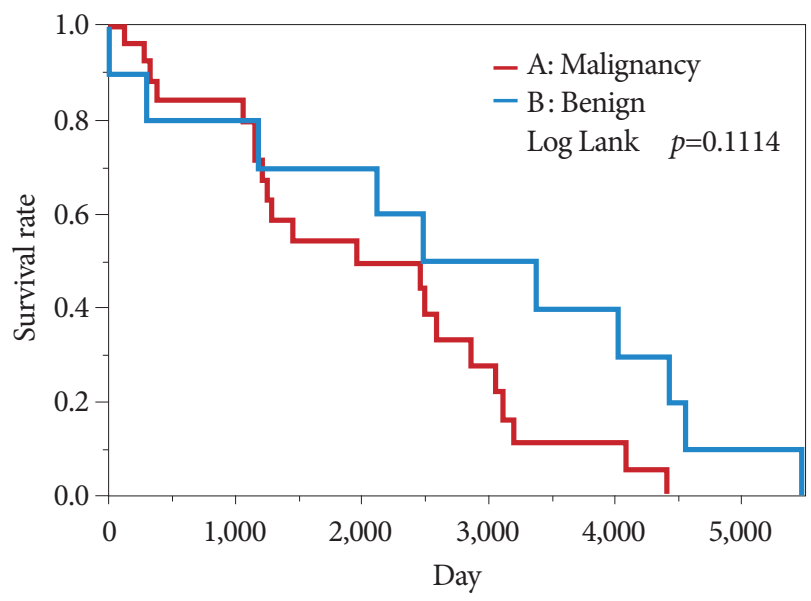

Fig. 9. Comparison of prognosis between the benign and malignant groups based on endoscopic diagnosis. 


\section{DISCUSSION}

In this single-center retrospective study, POPS was used to examine the endoscopic morphology and prognosis of IPMNs. The diagnostic sensitivity and specificity of cytology and biopsy were high in the Vil and Veg groups. With respect to prognosis based on preoperative endoscopic diagnosis, the benign group showed longer overall survival than the malignant group $(p=0.1114)$. In the study by El Hajj et al., ${ }^{30}$ postoperative pancreatitis was observed in approximately $4 \%$ of the patients; however, in our study, none of the patients had postoperative pancreatitis or other complications.

MD-IPMNs are characterized by ductal dilatation and intraductal papillary growth that can become malignant in $65-70 \%$ of the patients. ${ }^{31}$ Although initial diagnostic efforts rely on radiographic imaging and cystic fluid analysis, these modalities have limited sensitivity and they are often unable to differentiate benign from malignant lesions. ${ }^{1,32}$

Patients with MD-IPMNs have a 5-year survival rate of 31$54 \%$ after resection, ${ }^{32}$ which is a relatively good survival rate for pancreatic cancer, and surgery should be selected if malignant transformation is suspected. According to a follow-up observational report on nonresected BD-IPMNs, the frequency of progression to high-risk IPMNs was $0-0.3 \%$ during the follow-up period, and BD-IPMNs with no evidence of highrisk features had a favorable course; however, the incidence of pancreatic cancer with IPMN was relatively high, and the 5 -year survival rate of patients with pancreatic cancer with IPMN was $2.2-8.8 \%{ }^{4,33-35}$

Previous reports have shown the benefits of POPS and NBI with POPS. In 2002, Hara et al. ${ }^{27,28}$ effectively diagnosed benign and malignant lesions using a combination of POPS and intraductal ultrasonography (IDUS) for IPMNs and reported a large neoplastic lesion with a fish-egg-like appearance ${ }^{28}$ on endoscopy and a nodule $\geq 4 \mathrm{~mm}$ in size on IDUS, with an $88 \%$ malignancy rate. The 3-year cumulative survival rate of IPMN resection cases was $95 \%$; 1 of 40 cases had a positive surgical margin, and POPS was reported to be effective in determining the resection line. ${ }^{36}$ In our department, when using POPS alone, large, elevated lesions were often malignant, with a sensitivity of $85 \%$. The rate of accurate diagnosis was also high.

The prognosis of class I lesions is good, and follow-up is considered useful. Dye endoscopy is performed to detect small lesions in the digestive tract; however, POPS is not performed in the pancreatic duct, as it may cause acute pancreatitis. Therefore, it is difficult to diagnose flat lesions. However, the introduction of IEE with NBI enabled color and structural enhancement of the pancreatic duct and facilitated the detection of small, elevated lesions. Using NBI, it is possible to confirm the presence or absence of tumor vessels, which is considered to aid the diagnosis of benign and malignant lesions. It is impossible to insert a scope into the MPD in cases in which the MPD diameter is small; however, it is possible to distinguish benign and malignant lesions in cases of IPMNs in which the MPD diameter is large.

We examined the prognosis according to the morphologic classification obtained from the imaging diagnosis. In our study participants, Is cases had an increasing tendency, Isp cases were categorized as class IV or IIIb based on cytology, and Vil and Veg cases were malignant; hence, surgery was indicated.

IPMNs are usually diagnosed using ultrasonography and magnetic resonance cholangiopancreatography. For the diagnosis of benign and malignant conditions, endoscopic ultrasonography (Fukuoka guidelines for the management of $\mathrm{IPMNS}^{37}$ and IDUS ${ }^{28}$ are used to measure the pattern of cysts and the height of elevated lesions. ${ }^{38}$ Although these are effective for large protrusions, it may be challenging to visualize the border of flat lesions using IDUS or endoscopic ultrasonography.

In this study, cytology/biopsy performed at the time of POPS had sensitivity and specificity values of $85 \%$ and $87.5 \%$, respectively. Furthermore, if a scope can be inserted into the target site for diagnosing malignancy requiring surgical resection, it is considered useful in determining the resection line before surgery. With respect to the ability of POPS to determine the resection line, the margin was negative and positive in $95 \%$ and $5 \%$ of the patients, respectively. Hence, it was considered effective in diagnosing small lesions and in determining the surgical resection line, as also reported by Hara et al. ${ }^{28}$ When IPMNs were observed for a long time based on the endoscopic morphology of the tumor, the prognosis was considered poor in the case of large, elevated lesions. However, cases that were followed up without surgery were often benign, and the long-term prognosis of such cases may be good.

This study had some limitations. As it was a single-center study, the number of cases was small. Moreover, this was a retrospective study; therefore, multicenter, prospective studies with long-term follow-up periods are required to validate our findings.

Endoscopic diagnosis of IPMNs was performed by combining videoscopy and NBI, resulting in a clear image that helped in diagnosing blood vessel patterns and small ridges of the mucous membrane. Similarly, it was possible to diagnose benign and malignant IPMNs based on the type of protrusions. The ability to determine the surgical resection line was equally useful. Additionally, it was possible to estimate the long-term prognosis based on morphology. Strict follow-up was considered necessary in patients with small protrusions and tumor 
blood vessels. Nevertheless, further studies with a long-term follow-up are needed in the future.

Conflicts of Interest

The authors have no potential conflicts of interest.

Funding

None.

Acknowledgments

We thank the Department of Gastroenterology and Hepatology for their cooperation in conducting this study. We also thank the staff of the endoscopy and radiation departments for performing peroral pancreatoscopy. We would also like to express our gratitude to the professors at the Department of Surgical Pathology, Omori Hospital, for their help with tissue diagnosis and cytology.

Author Contributions

Conceptualization: Yui Kishimoto, Yoshinori Igarashi, Naoki Okano

Data curation and formal analysis: YK, Ken Ito, Kensuke Takuma, Seiichi Hara, Susumu Iwasaki, Kensuke Yoshimoto, Koji Watanabe, Yuuto Ymada, Hiroki Nakagawa

Writing-original draft: Yuusuke Kimura

Writing-review \& editing: YK, YI

\section{ORCID}

Yui Kishimoto

Naoki Okano

Ken Ito

Kensuke Takuma

Seiichi Hara

Susumu Iwasaki

Kensuke Yoshimoto

Yuto Yamada

Koji Watanabe

Yusuke Kimura

Hiroki Nakagawa

Yoshinori Igarashi

\begin{abstract}
https://orcid.org/0000-0001-5040-2938 https://orcid.org/0000-0002-9759-1374 https://orcid.org/0000-0001-5559-0990 https://orcid.org/0000-0003-3294-8990 https://orcid.org/0000-0003-4507-5412 https://orcid.org/0000-0001-8390-6032 https://orcid.org/0000-0002-7427-7059 https://orcid.org/0000-0002-8065-9037 https://orcid.org/0000-0001-7636-4001 https://orcid.org/0000-0002-5953-3843 https://orcid.org/0000-0001-9077-9289 https://orcid.org/0000-0002-9345-1649
\end{abstract}

\section{REFERENCES}

1. Tanaka M, Fernández-del Castillo $\mathrm{C}$, Adsay V, et al. International consensus guidelines 2012 for the management of IPMN and MCN of the pancreas. Pancreatology 2012;12:183-197.

2. Nagai K, Doi R, Kida A, et al. Intraductal papillary mucinous neoplasms of the pancreas: clinicopathologic characteristics and long-term follow-up after resection. World J Surg 2008;32:271-278; discussion 279280.

3. Hwang DW, Jang JY, Lee SE, Lim CS, Lee KU, Kim SW. Clinicopathologic analysis of surgically proven intraductal papillary mucinous neoplasms of the pancreas in SNUH: a 15-year experience at a single academic institution. Langenbecks Arch Surg 2012;397:93-102.

4. Crippa S, Fernández-Del Castillo C, Salvia R, et al. Mucin-producing neoplasms of the pancreas: an analysis of distinguishing clinical and epidemiologic characteristics. Clin Gastroenterol Hepatol 2010;8:213-219.

5. Schnelldorfer T, Sarr MG, Nagorney DM, et al. Experience with 208 resections for intraductal papillary mucinous neoplasm of the pancreas. Arch Surg 2008;143:639-646; discussion 646.

6. Nara S, Onaya H, Hiraoka N, et al. Preoperative evaluation of invasive and noninvasive intraductal papillary-mucinous neoplasms of the pancreas: clinical, radiological, and pathological analysis of 123 cases. Pancreas 2009;38:8-16.

7. Fritz S, Klauss M, Bergmann F, et al. Pancreatic main-duct involvement in branch-duct IPMNs: an underestimated risk. Ann Surg 2014;260:848855; discussion 855-856.

8. Robles-Medranda C, Valero M, Soria-Alcivar M, et al. Reliability and accuracy of a novel classification system using peroral cholangioscopy for the diagnosis of bile duct lesions. Endoscopy 2018;50:1059-1070.

9. Tajiri H. Diagnostic evaluation of pancreatic carcinoma and chronic pancreatitis by pancreatoscopy. Diagn Ther Endosc 1997;3:177-182.

10. Sugiyama M, Atomi $Y$, Saito M. Intraductal papillary tumors of the pancreas: evaluation with endoscopic ultrasonography. Gastrointest Endosc 1998:48:164-171.

11. Igarashi $\mathrm{Y}$, Ukita $\mathrm{T}$, Inoue $\mathrm{H}$, et al. Clinical evaluation of the peroral cholangioscopy using a new videoscope. Diagn Ther Endosc 1999;5:231237.

12. Kodama T, Sato H, Horii Y, et al. Pancreatoscopy for the next generation: development of the peroral electronic pancreatoscope system. Gastrointest Endosc 1999;49:366-371.

13. Kobayashi M. A study for the improvement of diagnostic ability of ultra-thin pancreatoscope: comparison of histological findings and application of image processing. Gastroenterological Endoscopy 1996; 38:2147-2159.

14. Uehara H, Nakaizumi A, Tatsuta M, et al. Diagnosis of carcinoma in situ of the pancreas by peroral pancreatoscopy and pancreatoscopic cytology. Cancer 1997;79:454-461.

15. Tajiri H, Kobayashi M, Ohtsu A, Ryu M, Yoshida S. Peroral pancreatoscopy for the diagnosis of pancreatic diseases. Pancreas 1998;16:408-412.

16. Jung M, Zipf A, Schoonbroodt D, Herrmann G, Caspary WF. Is pancreatoscopy of any benefit in clarifying the diagnosis of pancreatic duct lesions? Endoscopy 1998;30:273-280.

17. Kodama T, Koshitani T, Sato H, et al. Electronic pancreatoscopy for the diagnosis of pancreatic diseases. Am J Gastroenterol 2002;97:617-622.

18. Adler DG. Pancreatoscopy for pancreatic duct stones. Gastrointest Endosc 2014;79:208.

19. Yasuda K, Sakata M, Ueda M, Uno K, Nakajima M. The use of pancreatoscopy in the diagnosis of intraductal papillary mucinous tumor lesions of the pancreas. Clin Gastroenterol Hepatol 2005;3:S53-S57.

20. Igarashi Y, Okano N, Satou D, Miura T, Miki K. Peroral cholangioscopy using a new thinner videoscope (chf-B260). Dig Endosc 2005; 17:S63-S66.

21. Muto M, Sano Y, Fujii S, Ochiai A, Yoshida S. Endoscopic diagnosis of intraepithelial squamous neoplasia in head and neck and esophageal mucosal sites. Dig Endosc 2006;18:S2-S5.

22. Kodama T, Imamura $\mathrm{Y}$, Sato $\mathrm{H}$, et al. Feasibility study using a new small electronic pancreatoscope: description of findings in chronic pancreatitis. Endoscopy 2003;35:305-310.

23. Ringold DA, Shah RJ. Peroral pancreatoscopy in the diagnosis and management of intraductal papillary mucinous neoplasia and indeterminate pancreatic duct pathology. Gastrointest Endosc Clin N Am 2009;19:601613.

24. Parbhu SK, Siddiqui AA, Murphy M, et al. Efficacy, safety, and outcomes of endoscopic retrograde cholangiopancreatography with peroral pancreatoscopy: a multicenter experience. J Clin Gastroenterol 2017;51:e101-e105.

25. Yamao K, Ohashi K, Nakamura T, et al. Evaluation of various imaging methods in the differential diagnosis of intraductal papillary-mucinous tumor (IPMT) of the pancreas. Hepatogastroenterology 2001;48:962966.

26. Yamaguchi T, Hara T, Tsuyuguchi T, et al. Peroral pancreatoscopy in the diagnosis of mucin-producing tumors of the pancreas. Gastrointest Endosc 2000;52:67-73.

27. Mukai H, Yasuda K, Nakajima M. Differential diagnosis of mucin-producing tumors of the pancreas by intraductal ultrasonography and peroral pancreatoscopy. Endoscopy 1998;30:A99-A102. 
28. Hara T, Yamaguchi T, Ishihara T, et al. Diagnosis and patient management of intraductal papillary-mucinous tumor of the pancreas by using peroral pancreatoscopy and intraductal ultrasonography. Gastroenterology 2002;122:34-43.

29. Miura T, Igarashi Y, Okano N, Miki K, Okubo Y. Endoscopic diagnosis of intraductal papillary-mucinous neoplasm of the pancreas by means of peroral pancreatoscopy using a small-diameter videoscope and narrow-band imaging. Dig Endosc 2010;22:119-123.

30. El Hajj II, Brauer BC, Wani S, Fukami N, Attwell AR, Shah RJ. Role of per-oral pancreatoscopy in the evaluation of suspected pancreatic duct neoplasia: a 13-year U.S. single-center experience. Gastrointest Endosc 2017;85:737-745.

31. Lafemina J, Katabi N, Klimstra D, et al. Malignant progression in IPMN: a cohort analysis of patients initially selected for resection or observation. Ann Surg Oncol 2013;20:440-447.

32. Brugge WR, Lewandrowski K, Lee-Lewandrowski E, et al. Diagnosis of pancreatic cystic neoplasms: a report of the cooperative pancreatic cyst study. Gastroenterology 2004;126:1330-1336.

33. Uehara H, Nakaizumi A, Ishikawa O, et al. Development of ductal carcinoma of the pancreas during follow-up of branch duct intraductal papillary mucinous neoplasm of the pancreas. Gut 2008;57:1561-1565.

34. Tanno S, Nakano Y, Koizumi K, et al. Pancreatic ductal adenocarcinomas in long-term follow-up patients with branch duct intraductal papillary mucinous neoplasms. Pancreas 2010;39:36-40.

35. Kamata K, Kitano M, Kudo M, et al. Value of EUS in early detection of pancreatic ductal adenocarcinomas in patients with intraductal papillary mucinous neoplasms. Endoscopy 2014;46:22-29.

36. Date K, Ohtsuka T, Nakamura S, et al. Surveillance of patients with intraductal papillary mucinous neoplasm with and without pancreatectomy with special reference to the incidence of concomitant pancreatic ductal adenocarcinoma. Surgery 2018;163:291-299.

37. Tanaka M, Fernández-Del Castillo C, Kamisawa T, et al. Revisions of international consensus Fukuoka guidelines for the management of IPMN of the pancreas. Pancreatology 2017;17:738-753.

38. Igarashi Y, Tada T, Shimura J, et al. Endosonographic diagnosis of intraductal papillary-mucinous pancreatic tumors by intraductal ultrasonography. Dig Endosc 2003;15:196-199. 